\title{
1 Biomass of a trophic level increases with maximum body size, but less than proportionally
}

2 Henrique C. Giacomini

3 Ontario Ministry of Natural Resources and Forestry, 2140 East Bank Drive, Peterborough, ON, Canada, $4 \quad$ K9J $7 B 8$ concluded that large-sized species contribute disproportionately to several ecosystem functions. One of

9 their key predictions is that total biomass of animals in a trophic level ( $M_{\text {Tot }}$, using their notation) should increase more than proportionally with its maximum body size $\left(m_{\max }\right)$, following the relationship $M_{\text {Tot }} \propto m_{\max }^{5 / 4}$. Here I argue that this superlinear scaling results from an incorrect representation of

12 the individual size distribution and that the exponent should be $1 / 4$, implying a sublinear scaling. The same reasoning applies to total energy flux or metabolism $B_{T o t}$, which should be invariant to maximum size according to the energetic equivalence and perfect compensatory responses entailed by MST. the product of individual mass $m$ by its density function $f(m)$, also known as size spectrum or individual

17 size distribution:

$$
M_{T o t}=\int_{m_{0}}^{m_{\max }} m f(m) d m
$$


21 unit space (denoted here by $N_{\text {Tot }}$ ). This is also a necessary condition for $M_{\text {Tot }}$ in equation (1) to be 22 measured in the correct scale, in total mass per unit space. number of individuals per unit space, so it is not suitable as a density funtion to calculate biomass in equation (1). The abundance spectrum of a trophic level can be represented as a function of the 30 interval's maximum size as $N_{T o t}=\delta m_{\max }{ }^{-3 / 4}$ (which reference size is used, whether maximum,

31 minimum or a mid-point, does not change the exponent ${ }^{7}$ ). The relationship between $N_{\text {Tot }}$ and $f(m)$ can 32 be thus expressed as:

$N_{T o t}=\int_{m_{0}}^{m_{\max }} f(m) d m=\int_{m_{\max } / a}^{m_{\max }} c m^{-\epsilon} d m=\delta m_{\max }{ }^{-3 / 4}$

where $a>1$ is a constant defining the ratio $m_{\max } / m_{0}$, or the logarithmic range of the size interval; and $\delta$ is a coefficient given by $\delta=4 c\left(a^{3 / 4}-1\right) / 3$. Most importantly, the exponent satisfying equation (2) is $35-\epsilon=-7 / 4$, one unit lower than the value used by Enquist et al. Similar demonstrations can be found in refs. 7-8.

The value of $\epsilon$ can be derived from more fundamental principles of MST if we assume a constant resource supply rate $\left(U_{T o t} \text {, in mass per time per unit space }\right)^{3}$. At equilibrium, the total metabolism or energy flux of a trophic level, $B_{T o t}$, should be equal to their shared $J_{T o t}$ (assuming further that $J_{T o t}$ corresponds to assimilated energy), so that biomass remains constant in time: 
$\frac{d M_{T o t}}{d t}=J_{T o t}-B_{T o t}=0$

41 The total metabolism is equal to individual metabolism integrated over the size spectrum $f(m)$.

42 Individual metabolism is expected to scale as $\propto m^{3 / 4}$ (ref. 3), so equation (3) can be expressed as:

$J_{\text {Tot }}=B_{\text {Tot }} \propto \int_{m_{\max } / a}^{m_{\max }} m^{3 / 4} \mathrm{~cm}^{-\epsilon} d m \propto m_{\max }{ }^{7 / 4-\epsilon}$

43 As $J_{T o t}$ is invariant with respect to size $\left(J_{\text {Tot }} \propto m^{0}\right)$, the equilibrium condition requires that $\epsilon=7 / 4$.

The assumption of constant resource supply underlies the energetic equivalence rule or

hypothesis ${ }^{4}$ and explains the commonly observed scaling of $N_{T o t}$ with an exponent $-3 / 4$ within a trophic

level (or, more generally, with the reciprocal of individual metabolism's). The reasons for energetic equivalence are still a debated topic, and the hypothesis have found mixed empirical support (e.g., refs.

$\Lambda=\frac{\gamma_{i} m^{3 / 4}-\sum_{k} \gamma_{k} m^{3 / 4}}{\gamma_{i} m^{3 / 4}}$

52 where $\gamma_{i}$ is the coefficient for energy input (e.g., ingestion), and $\gamma_{k}$ are the coefficients representing all

53 processes leading to energy losses (i.e., not used by the next trophic level), including respiration, 
57 constant $J_{T o t}$ and $B_{T o t}$ ) regardless of which body sizes characterize the trophic level, and the resulting

58 size spectrum scales as $f(m) \propto m^{-7 / 4}$ (equation 4), implying $N_{T o t} \propto m^{-3 / 4}$ (equation 2).

$$
M_{T o t}=\int_{m_{0}}^{m_{\max }} m c m^{-7 / 4} d m=4 c\left(m_{\max }^{1 / 4}-m_{0}^{1 / 4}\right) \approx 4 c m_{\max }^{1 / 4}
$$

where the resulting power function is an approximation for small $m_{0}$. It predicts an increase of biomass

61 with maximum body size, but in a sublinear way.

It is important to recognize that the increase in biomass predicted by MST in equation (6) does not involve changes in ecological function. It results purely from the fact that larger organisms have slower turnover rates, which leads to greater biomass accumulation. From a community perspective, it implies perfect compensatory responses to species losses, as total resource utilization rate remains unchanged. Energy equivalence, constant trophic efficiency, and power-law size spectra are intrinsically related aspects of MST and all result from a common scaling of energetic processes. While there may be reasons for common scaling to result from long-term evolutionary processes ${ }^{11}$ or broad-scale macroecological averaging, many real cases of ecological interest involve transient short- or mid-term changes that deviate from MST assumptions. For instance, trophic interactions are generally sizestructured, so smaller predators in a trophic level cannot immediately compensate for the loss of large ones. Due to the increased proportion of uneaten prey, trophic efficiency (equation 5) would decrease nonlinearly with size, preventing analytical solutions in the form of simple power functions and limiting the scope of MST. In those cases, MST predictions are not expected to match empirical data or simulations from more realistic models. One example is the $18 \%$ decline in total heterotrophic metabolism that resulted from megaherbivore losses in the General Ecosystem Model simulations by Enquist et al., in contrast with the MST expectation of no change. Nonetheless, MST still serves as a 
usefull baseline for comparison, one that controls for purely energetic processes but leaves out other

79 important factors such as specialized ecological interactions and transient dynamics.

80

81

82

83

84

85

86

87

88

89

90

91

92

\section{References}

1. Enquist, B.J., Abraham, A.J., Harfoot, M.B., Malhi, Y. \& Doughty, C.E. The megabiota are disproportionately important for biosphere functioning. Nature Communications, 11(1) 1-11 (2020).

2. Andersen, K.H. \& Beyer, J.E. Asymptotic size determines species abundance in the marine size spectrum. The American Naturalist, 168(1) 54-61 (2006).

3. Brown, J.H., Gillooly, J.F., Allen, A.P., Savage, V.M. \& West, G.B. Toward a metabolic theory of ecology. Ecology, 85(7) 1771-1789 (2004).

4. White, E.P., Ernest, S.M., Kerkhoff, A.J. \& Enquist, B.J. Relationships between body size and abundance in ecology. Trends in Ecology \& Evolution, 22(6) 323-330 (2007).

5. Sprules, W.G. \& Barth, L.E. Surfing the biomass size spectrum: some remarks on history, theory, and application. Can. J. Fish.Aquat. Sci. 73(4) 477-495 (2016).

6. Jennings, S. \& Mackinson, S. Abundance-body mass relationships in size-structured food webs. Ecology Letters, 6(11) 971-974 (2003).

7. Blanco, J.M., Echevarría, F. \& García, C.M. Dealing with size-spectra: some conceptual and mathematical problems. Sci. Mar. 58: 17-29 (1994).

8. Reuman, D.C., Mulder, C., Raffaelli, D. \& Cohen, J.E. Three allometric relations of population density to body mass: theoretical integration and empirical tests in 149 food webs. Ecology Letters, 11(11) $1216-1228$ (2008).

9. Isaac, N. J., Storch, D. \& Carbone, C. Taxonomic variation in size-density relationships challenges the notion of energy equivalence. Biology Letters 7:615-618 (2011). 
101 10. Hatton, I.A., Dobson, A.P., Storch, D., Galbraith, E.D. \& Loreau, M. Linking scaling laws across eukaryotes. Proceedings of the National Academy of Sciences, 116(43) 21616-21622 (2019).

103 11. Hin, V. \& de Roos, A.M. Evolution of size-dependent intraspecific competition predicts body size scaling of metabolic rate. Functional Ecology, 33(3), 479-490 (2019). 